\title{
Reservoir simulation to seismic: an approach for time lapse analysis
}

Maurício Laurindo de Matos ${ }^{1}$, Jadson Muniz de Oliveira ${ }^{1}$, Allan Peixoto de Franco ${ }^{1}$, Lucas Gabriel Silva de Aguiar ${ }^{1}$, Rodrigo da Silva Canário ${ }^{1}$ and Fernando Sergio de Moraes ${ }^{1},{ }^{1}$ Grupo de Inferência de Reservatório - GIR/LENEP/UENF

Copyright 2021, SBGf - Sociedade Brasileira de Geofísica

This paper was prepared for presentation during the $17^{\text {th }}$ International Congress of the Brazilian Geophysical Society held in Rio de Janeiro, Brazil, 16-19 August 2021.

Contents of this paper were reviewed by the Technical Committee of the $17^{\text {th }}$ International Congress of the Brazilian Geophysical Society and do not necessarily represent any position of the SBGf, its officers or members. Electronic reproduction or storage of any part of this paper for commercial purposes without the written consent of the Brazilian Geophysical Society is prohibited.

\begin{abstract}
Reservoir simulation and time-lapse seismic provide complementary information. These two techniques combined can help to improve the knowledge about the reservoir behavior. A valuable tool employed in reservoir management is the simulation to seismic modeling, a forward technique used to generate synthetic seismic response from a reservoir model. In this work, we present a new simtoseis approach, using seismic AVO inversion information with reservoir simulator results to create mineral bulk modulus model and perform the petroelastic modeling. We employ machine learning techniques to convert spatial information between the seismic and simulator domains. To evaluate the synthetic and real seismic data, the same sections are plotted and anomalies are interpreted along with saturation and pressure maps. The described workflow supports many applications, such as AVO analysis, seismic time-lapse survey design, reservoir characterization and monitoring.
\end{abstract}

\section{Introduction}

Reservoir management is a dynamic process that goes through the entire life cycle and requires the effective integration of people, technologies, and data to maximize profit by increasing oil and gas production while minimizing operational costs. A valuable tool employed in reservoir management is the production performance forecast through flow simulation; these techniques can quantify the fluid movements for a reservoir model.

Reservoir Model is a three-dimensional representation of a reservoir based on its petrophysical, geological and geophysical properties. A model is formed by cells distributed respecting the heterogeneities of the reservoir, with each cell containing a set of static and dynamic properties. During flow simulation, engineers aim to predict the future behavior of the field over a specific time interval. This prediction includes production volumes, pressure, and saturation changes. The ability to forecast potential scenarios with reasonable precision can show the best resource allocation and development plans for each prospect, considering the economic setting.

Time-lapse (4D) seismic is the seismic acquisition over the field's productive life. A critical concern about the data is quality and repeatability. This surveillance technique allows geoscientists to map the elastic changes in the reservoir, using differences between two or more surveys. Then, inferences about the field's production effects and drainage pattern help to improve geological knowledge and update/calibrate the reservoir model.

Reservoir simulation and time-lapse seismic provide complementary information. Therefore, these two techniques combined can help to improve the knowledge about the reservoir behavior. Simulator to seismic modelling (simtoseis) bridges the gap between the flow simulation model and the real seismic, and opens a new platform for taking 4D seismic data into the core of the reservoir surveillance and management plan (Ahmed 2014).

Simtoseis is a forward modeling technique used to predict/generate synthetic seismic response from a reservoir model. It is composed of two key processes (Fig. 1): first, the results of flow simulation are converted to elastic properties through the petroelastic model (PEM), and second, seismic modeling is employed to generate the synthetic seismic (Ahmed, 2011).

There are several applications for simtoseis in reservoir characterization; it can test the consistency of a 3D geological static model, or to evaluate the historymatched simulation (dynamic) model, comparing the real time lapse anomalies with simulated results. The value of integrating static and dynamic data for a more accurate reservoir characterization, and consequently building a reliable reservoir models, are described by Tabatabaei (2014). Besides, simulation to seismic modeling is essential to investigate the effects of saturation change in the seismic response, and thus, determine the appropriate time shift in the production history to shoot the monitoring survey. 
RESERVOIR SIMULATION TO SEISMIC, AN APPROACH FOR TIME LAPSE ANALYSIS

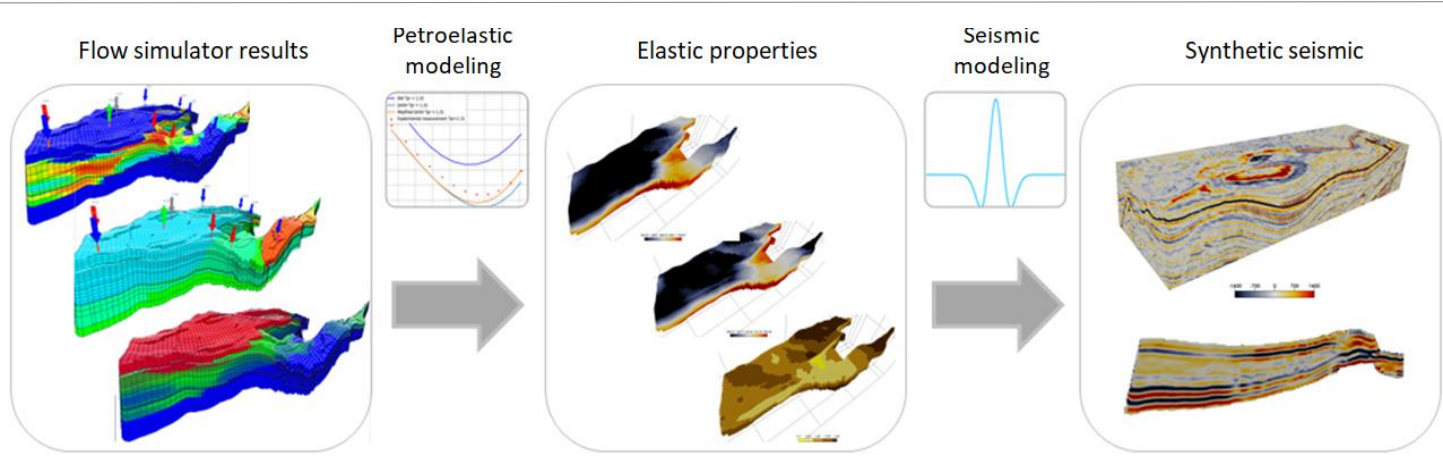

Figure 1 - Reservoir simulation to seismic standard chart.

In this work, we present a new simtoseis approach, using seismic AVO inversion information (velocity $p$, velocity $s$ and density) to create mineral bulk modulus model (in simulator domain) and perform the petroelastic modeling. We employ an alternative method for calculating the physical properties of hydrocarbons and use machine learning techniques to convert spatial information between the seismic and simulator domains. The entire workflow is developed in an open source environment. The modeling is applied in the Norne field case study between the years 2001 and 2006 to validate the method. Norne benchmark is hosted and supported by the 10 Center at NTNU and available for universities and research centers.

\section{Method}

The proposed approach includes five subprocesses (Fig. 2) and starts running the simulation model (1) to extract the static and the dynamic properties at the selected time steps. To perform the PEM, the seismic inversion data at initial time must be converted from the seismic domain to simulator domain (2). Then, PEM with fluid substitution (3) is performed in the simulator domain, converting the reservoir properties to elastic properties and updating the elastic properties from the base time to the selected time. After that, the petroelastic results must be converted (4) to the seismic grid where the seismic modeling is performed. Finally, a seismic modeling (5) is employed to generate the synthetic seismic.

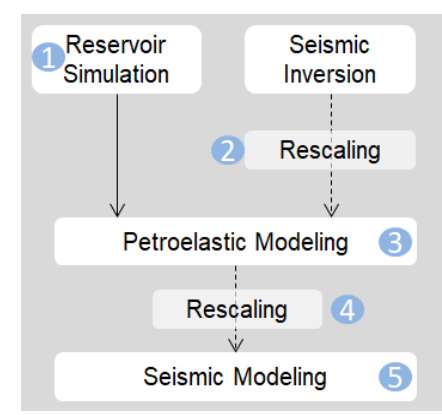

Figure 2- Simtoseis approach.

\section{Reservoir Simulation}

The Norne simulation model is a three-phase, corner point grid, black-oil model comprising 113.344 cells, distributed in 22 layers between the depths of $2439 \mathrm{~m}$ and $3090 \mathrm{~m}$. The average grid block size is $100 \mathrm{~m} \times 95 \mathrm{~m} \times 10$ $\mathrm{m}$. The simulation is carried out between 1997 and 2006 . A set of static and dynamic properties for the selected time-steps are made available for the grid conversion and petroelastic modeling subprocesses.

\section{Seismic to simulator domain conversion}

The proposed PEM is performed on the simulation grid scale and aims to estimate the elastic moduli of the saturated rock at the time of interest (monitor time), based on the conditions at the base time extracted from the elastic seismic inversion. For this, the elastic properties from inversion data on the seismic cube scale must be assigned to each cell in the reservoir simulation model.

The seismic grid is a regularly spaced mesh ( $x, y$, and $z$ ), with $z$ representing either depth or time. In turn, the reservoir simulation grid comprises a set of irregularshaped cells, in which each cell is described through the coordinates of its eight corners. The elastic properties of each sample in the seismic cube domain are interpolated for each cell of the simulation grid, represented by the $x, y$ and $z$ coordinates of their respective centers.

To perform this task, we use the ExtraTrees Regressor method. This method is a multiple decision tree algorithm that combines the predictions from a selected number of unpruned trees constructed by the algorithm from the training dataset. The major difference from other ensembles methods is the total randomness used to define the random selection of features and their respective threshold in node splitting (Geurts et al., 2006). The randomness of trees tends to increase the accuracy since the error in the prediction in different trees tends to be uncorrelated (Breiman, 2001).

\section{Petroelastic modeling and fluid substitution}


The petroelastic modeling step employs conventional fluid substitution based on Gassmann's equation see e.g., Kumar (2006) and Smith \& Sondergeld (2003), with MacBeth (2004) equations and empirical correlations for dry-rock characterization. We can divide the entire process into seven steps:

3.1. Applying the inversion cubes, we calculate the elastic moduli of the saturated rock for the base time:

$\mathrm{K}_{\mathrm{sat}}=\rho\left(\mathrm{V}_{\mathrm{p}}^{2}-\frac{4}{3} \mathrm{~V}_{\mathrm{s}}^{2}\right)$;

Eq. 1

$\mu_{\mathrm{sat}}=\rho V_{\mathrm{s}}^{2}$.

Eq. 2

3.2. Prior to performing a fluid substitution, we must know the bulk modulus and density of the in situ pore-filling fluid in the base time. Because there typically are two or more fluid phases occupying the pore space of a reservoir rock, we must calculate a bulk modulus and density of the individual members and then mix the phases according to the saturation state considered (uniform or patchy saturation). The maps of dynamic (e.g., saturations, pressure, and GOR) and static (e.g., porosity, and NTG) properties used in this stage are obtained from reservoir simulation forecasts.

To estimate the elastic properties of each fluid phase, we propose two methodologies: the well-known Batzle \& Wang (1992) equations and an alternative formulation proposed by Carvalho \& Moraes (2020) where individuals guidelines for computing acoustic velocity for oil and gas are presented in a series of steps that combine state equations and empirical correlations, using as input: pressure, temperature, molecular weight and gas specific gravity.

3.3. Traditionally, the calculation of the mineral mixture bulk modulus is performed through a weighted average between the volumetric fraction and elastic modulus of each constituent. Because of the variability of the mineral content of the rocks and the difficulty of obtaining precisely the fraction of each constituent, this method may not represent the heterogeneities of a real reservoir. Furthermore, clay/shale parameters are widely variable and reservoir dependent. To address this problem, we combine the Gassmann (1952) equation and the Nur et al. (1995) empirical correlation to estimate the mineral bulk modulus of the reservoir model.

First, we replace the dry rock bulk modulus in the Gassmann equation with the Nur correlation (Eq. 3), and then, we explain the Gassmann equation for the mineral mixture bulk modulus (Eq. 5). Critical porosity can be obtained by the critical porosity value of each constituent weighted by its volume.

$$
\begin{array}{ll}
\mathrm{K}_{\text {frame }}=\mathrm{K}_{\min }\left(1-\frac{\phi}{\phi_{\text {crit }}}\right), & \text { Eq. } 3 \\
\mathrm{~B}=(1+\phi) \mathrm{K}_{\mathrm{fl}}-\left(\mathrm{K}_{\text {sat }}+\mathrm{K}_{\mathrm{fl}}\right) \phi_{\text {crit }}, & \text { Eq. } 4 \\
\mathrm{~K}_{\text {min }}=\frac{-\mathrm{B}+\sqrt{\mathrm{B}^{2}+4\left(1-\phi_{\text {crit }}\right)\left(\phi_{\text {crit }}-\phi\right) \mathrm{K}_{\mathrm{fl}} \mathrm{K}_{\text {sat }}}}{2\left(\phi_{\text {crit }}-\phi\right)} & \text { Eq. } 5
\end{array}
$$

3.4. After determining the mineral mixture bulk modulus, we can estimate the dry rock bulk modulus using Eq. 3. The shear modulus for dry rock is equal to the shear modulus for saturated rock since this property is insensitive to fluids.

3.5. Variations of rock-frame properties with applied effective stress are a critical ingredient in assessing the feasibility of $4 \mathrm{D}$ monitoring projects and in the inversion for dynamic reservoir properties from seismic (MacBeth, 2004). To update the dry rock properties for the time of interest, MacBeth (2004) proposes a formula based on rock-dependent parameters and the effective pressure to describe the sensitivity of elastic moduli for a sandstone rock frame (Eq. 6 and Eq. 7). The parameters are calibrated through laboratory analysis and characterized by the porosity and compacting of the rock under study.

$$
\begin{aligned}
& \mathrm{K}_{\text {frame_2 }}=\mathrm{K}_{\text {frame } \_1} \frac{1+\mathrm{E}_{\mathrm{k}} \mathrm{e}^{\left(\frac{-\mathrm{P}_{\text {eff_1 }}}{\mathrm{P}_{\mathrm{k}}}\right)}}{1+\mathrm{E}_{\mathrm{k}} \mathrm{e}^{\left(\frac{-\mathrm{P}_{\text {eff__ }}}{\mathrm{P}_{\mathrm{k}}}\right)},} \\
& \mu_{\text {frame } \_2}=\mu_{\text {frame } \_1} \frac{1+\mathrm{E}_{\mu} \mathrm{e}^{\left(\frac{-\mathrm{P}_{\text {eff_1 }}}{\mathrm{P}_{\mu}}\right)}}{1+\mathrm{E}_{\mu} \mathrm{e}^{\left(\frac{-\mathrm{P}_{\text {eff_2 }}}{\mathrm{P}_{\mu}}\right)}} .
\end{aligned}
$$

3.6. To estimate the elastic moduli of the fluid mixture for the time of interest, we employ the same process showed in step 3.2.

3.7. Finally, we calculate the saturated rock bulk modulus through the Gassmann equation (Eq. 8). The shear modulus will be the same as for dry rock (Eq. 9).

$\mathrm{K}_{\mathrm{sat} \_2}=\mathrm{K}_{\text {frame } \_2}+\frac{\left(1-\frac{\mathrm{K}_{\text {frame } \_2}}{\mathrm{~K}_{\min }}\right)^{2}}{\frac{\phi}{\mathrm{K}_{\mathrm{fl} \_2}}+\frac{(1-\phi)}{\mathrm{K}_{\min }}-\frac{\mathrm{K}_{\text {frame } 2}}{\mathrm{~K}_{\min }{ }^{2}}}$,

$\mu_{\text {sat } \_2}=\mu_{\text {frame_2 }}$.

We can computed the density by the following average:

$\rho_{\text {sat } \_2}=\phi \rho_{\mathrm{fl} \_2}+(1-\phi) \rho_{\min } . \quad$ Eq. 10 
We can rearrange the equations 1 and 2 to get velocities $\mathrm{P}$ and $\mathrm{S}$ for the saturated rock. Fig. 3 shows the velocity model of the petroelastic modeling performed in this study.

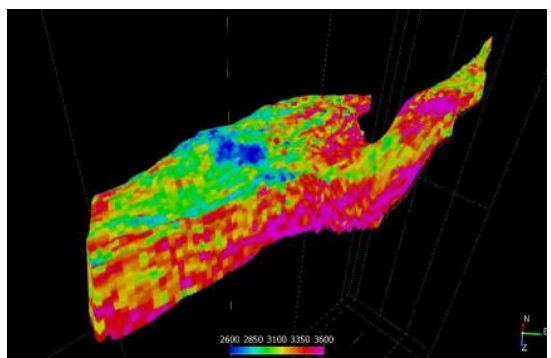

Figure 3 - Vp from petroelastic model.

\section{Simulator domain to Seismic domain conversion}

To perform the seismic modeling, we must convert the results of the petroelastic modeling to the seismic domain. The elastic properties in each cell of the reservoir simulation model are rescaled for each sample in each trace on the seismic scale using two techniques: extraction of pseudo-logs by computational geometry adapted from Amini (2014) and by machine learning through the ExtraTrees Regressor.

\subsection{Pseudo-log extraction}

From each CMP, a vertical pseudo-log intercepts the reservoir grid, capturing the depths of interfaces between the cells. We assign to each seismic sample between the interfaces the property values for that cell. Fig. 4 presents a conceptual example of extracting a pseudo-log.

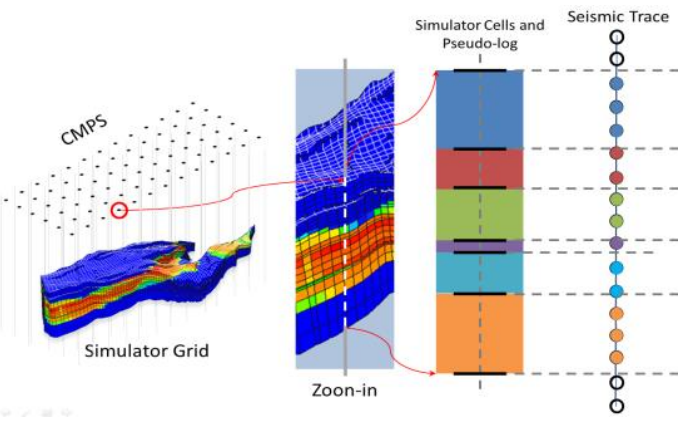

Figure 4 - The property value in the cells (different colors) is assigned to the seismic samples, considering the interfaces of each cell. This technique considers complex reservoir geometric features like pinch-out, folds and oblique pillars. Since the technique seeks to capture the edges of each cell, the result exhibits a blocked aspect, similar to the reservoir simulator model but in seismic sampling.

\subsection{ExtraTrees Regressor}

The pseudo-logs extraction can require significant computational time depending on the number of CMPs and seismic sampling. In addition, the blocked aspect may be undesired. As an alternative, we propose to apply the ExtraTrees regressor similarly to the process presented in stage 2 but in the opposite direction. The property in the reservoir simulation model, represented by the $x, y$ and $z$ coordinates of the center of each cell is interpolated for the seismic domain, represented by the coordinates of each seismic sample. The result shows a smoothed appearance. Fig. 5 presents the conversions performed by both methods.

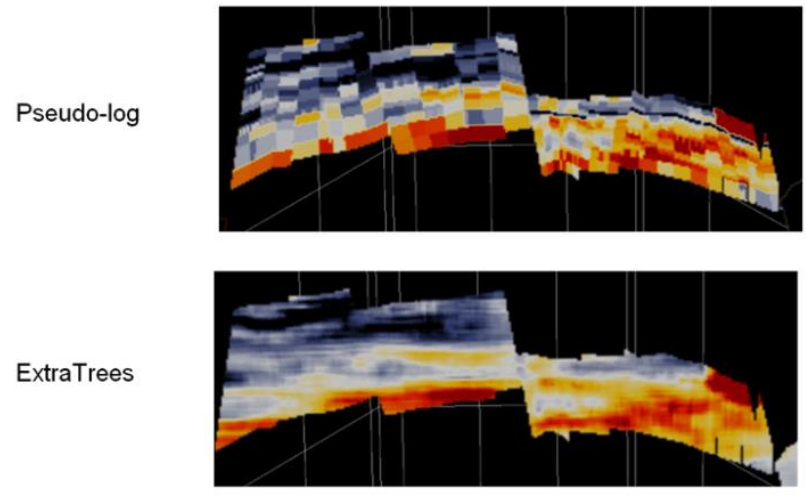

Figure 5 - Domain conversion by Pseudo-log extraction and Extratrees Regressor.

\section{Seismic modeling.}

In order to build seismic synthetic data the convolutional modeling has been used. For a modeling that accounts for multiples, mode conversion and AVO effect, the reflectivity method described by Fuchs \& Muller can be used as well. To apply this strategy the velocity model above the reservoir is needed, so a smooth velocity model (from seismic processing) has been used and the values inside the reservoir have been replaced by the simulated velocities and thus, completing the velocity grid.

\section{Results}

To show our preliminary results, we choose 2001 to 2006 time lapse because the longest period between seismic surveys and the reservoir would indicate larger variations. Fig. 6 shows the amplitude anomalies for the real data $(A)$ and synthetic (B) followed by water saturation increase (C) and pore pressure difference (D) expected from the simulated model. We trace a horizon along the inferred washed zone using the hardening anomalies trend observed in real amplitude difference cube and select the main softening ( $\mathrm{S}$ - red) and hardening $(\mathrm{H}$ - blue) anomalies to discuss. 

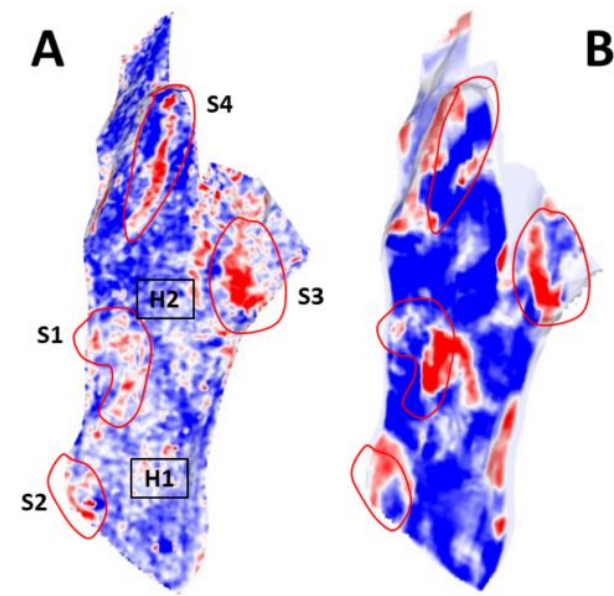

B
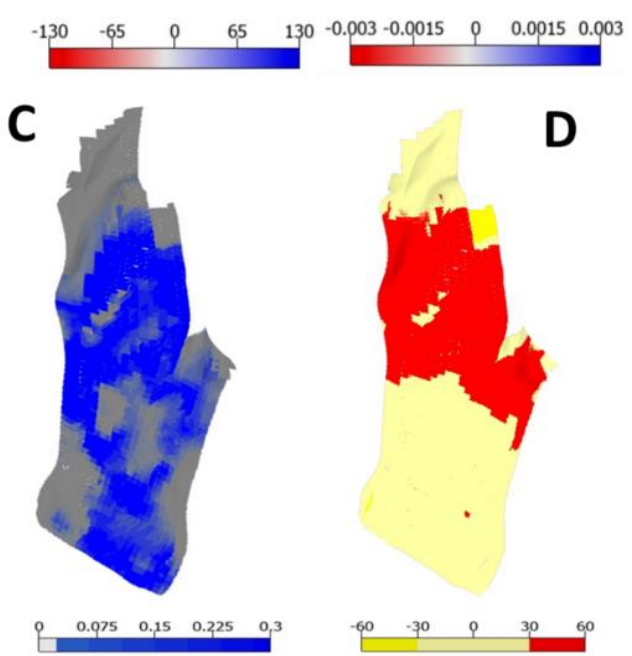

Figure 6 - Amplitude anomalies for the real data $(A)$ and synthetic $(B)$. Water saturation increase $(C)$ and pore pressure difference $(D)$.

To assist our interpretation, Fig. 7 shows a fault map from simulation model (left) and the producer and injector wells (right), red and blue dots respectively. The Norne field can be separated in two compartments: the Main Structure (C, D and E-segment) and the North-East Segment (G-segment). We focus our analysis on the Main Structure.

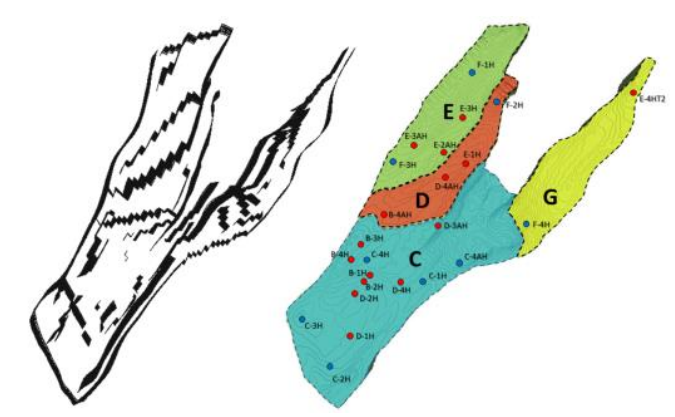

Figure 7- Fault map (left) and Norne segments (right).
S1 in segment $\mathrm{C}$ shows a softening anomaly interpreted as injection of gas in well $\mathrm{C} 4 \mathrm{H}$. The interpretation can identify a flow barrier that was correctly inferred during the reservoir flux model building (see Fig. 7 left).

$\mathrm{S} 2$ in segment $\mathrm{C}$ has a gas injecting well $\mathrm{C} 3 \mathrm{H}$. The anomalies are a little displaced from the expected position and have less extension than proposed by the synthetic results. It is possible to identify the spatial restriction though.

S3 correlates with a topographic low cote. The well C4AH was a water and gas injector and it was placed there to provide pressure support and enhance the oil sweep. The increase in pore pressure may have caused a drop in the effective pressure and consequently producing a softening anomaly.

S4 is the structural barrier or fault between segments $D$ and $\mathrm{E}$. It is possible to identify a spatial displacement between modeled and synthetic results, probably because of wavelet resolution used to build the synthetic case.

Analyzing the cross-section (Fig. 8) it is possible to identify a better resolution for the synthetic case (upper) and a little displacement upward for the hardening anomaly over the reservoir if compared to the real data (lower). Black spots indicate where water saturation has increased. A good match between synthetic and real model has been achieved. The green line indicates the intersection between the cross section and the interpreted horizon.

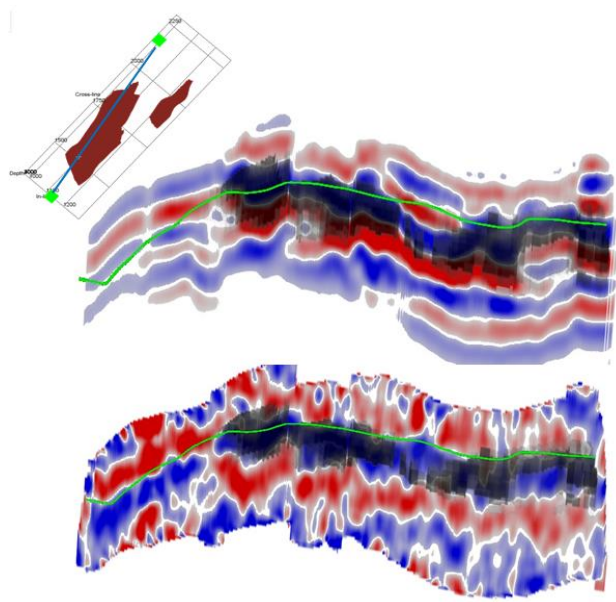

Figure 8 - Synthetic data (upper) and real data (lower) cross-sections with water saturation increase (black spots).

$\mathrm{H} 1$ and $\mathrm{H} 2$ inside segment $\mathrm{C}$ and $\mathrm{D}$ correlates with production zones and regions where water has replaced oil, probably caused the hardening anomalies and indicating swept zones. 
RESERVOIR SIMULATION TO SEISMIC, AN APPROACH FOR TIME LAPSE ANALYSIS

Hopefully, the reader can correlate hardening anomalies with the water saturation increase map. The pressure anomaly in segment $\mathrm{C}$ east limit, observed as softening anomalies in the synthetic case, is not presented in real results due to a horizontal shift. In that region, it was not possible to infer the flow barrier proposed in the simulator model.

\section{Conclusions}

To evaluate the synthetic and real model, the same sections are plotted with water saturation, faults and pressure maps. The result shows similarity between the models, although in some regions the anomalies were displaced.

For proper simtoseis modeling, it is fundamental to evaluate the real seismic data quality. Reliable 4D inferences are difficult to achieve and specific processing strategies are needed to generate results as similar as possible, leaving just anomalies caused by reservoir changes. Also, it is important to understand the resolution limits for both spatial (wavelet frequency) and amplitudes (reflectivity).

Accurate parameters for petroelastic modeling and fluid replacement are essential to obtain a realistic match between synthetic and real data. Norne is a well-known field, which contributes with correct inputs for the petroelastic model. The proposed two-stage approach dispenses the need for elastic modulus of rock-forming minerals but directly depends on an accurate seismic elastic inversion.

Considering the different geometric features present in a reservoir model is an important step in this simtoseis workflow. Domain conversion by pseudo-log extraction works properly for the data under study. For a lower computational cost, the ExtraTrees regressor can be used.

The described workflow supports many applications, such as AVO analysis, seismic time-lapse survey design, reservoir characterization and monitoring.

\section{Acknowledgments}

We would like to thank: Petrobras for funding the R\&D project registered as "Desenvolvimento de fluxos de trabalho e metodologias para processamento e interpretação quantitativa de dados sísmicos 3D e 4D"; Equinor for providing the Norne dataset used during this study; dGB Earth Sciences for the open-source seismic interpretation software OpendTect, and the Open Porous
Media initiative contributors for making their software available for reservoir simulator visualization.

\section{References}

Amini, H., Stanford Exploration Project Report 2014, A pragmatic approach to simulator-to-seismic modelling for $4 d$ seismic interpretation: Ph.D. thesis, Heriot-Watt University.

Batzle, M., and Wang, Z., 1992, Seismic properties of pore fluids: GEOPHYSICS, 57, no. 11, 1396-1408.

Breiman, L. (2001). Random forests. Machine learning, 45(1), 5-32.

Carvalho, R. R., \& Moraes, F. S., 2018. Evaluation of an Alternative Formulation for Computing Seismic Properties of Hydrocarbon Fluids. In 80th EAGE Conference and Exhibition 2018.

Dadashpour M 2009 Reservoir Characterization Using Production Data and Time-Lapse Seismic Data, PhD thesis, Norwegian University of Science and Technology (NTNU).

Fuchs, K., and Muller," G., 1971, Computation of synthetic seismograms with the reflectivity method and comparison with observations: Geophysical Journal of the Royal Astronomical Society, 23, no. 4, 417-433.

Gassmann, F., 1951. Uber die elastizitat poroser medien. Vierteljahrsschrift der Naturforschenden Gesellschaft in Zurich, 96, 1-23.

Geurts, P., Ernst, D., \& Wehenkel, L. (2006). Extremely randomized trees. Machine learning, 63(1), 3-42.

Kumar, D., 2006. A tutorial on Gassmann fluid substitution: formulation, algorithm and Matlab code. matrix, 2(1).

MacBeth, C., 2004, Classification for the pressure sensitivity properties of a sandstone rock frame: Geophysics, 69, no. 2, 497-510.

Moraes, F., and MacBeth, C., 2005, Saturation and pressure inference from velocities and density:, in 9th International Congress of the Brazilian Geophysical Society \&amp; EXPOGEF, Salvador, Bahia, Brazil, 1114 September 2005, 1297-1300.

Nur, A. M., Mavko, G., Dvorkin, J., and Gal, D., 1995, Critical porosity: The key to relating physical properties to porosity in rocks:, in SEG Technical Program Expanded Abstracts 1995, 878-881.

Smith, T. M., Sondergeld, C. H., and Rai, C. S., 2003, Gassmann fluid substitutions: A tutorial: GEOPHYSICS, 68, no. 2, 430-440. 